\title{
Delay and Its Time-Derivative Dependent Stable Criterion for Differential-Algebraic Systems
}

\author{
Hui Liu, Yucai Ding \\ School of Science, Southwest University of Science and Technology, Mianyang, China \\ Email: dycyer@163.com
}

Received 21 April 2016; accepted 21 June 2016; published 24 June 2016

Copyright @ 2016 by authors and Scientific Research Publishing Inc.

This work is licensed under the Creative Commons Attribution International License (CC BY). http://creativecommons.org/licenses/by/4.0/

c) (i) Open Access

\begin{abstract}
In this paper, the stable problem for differential-algebraic systems is investigated by a convex optimization approach. Based on the Lyapunov functional method and the delay partitioning approach, some delay and its time-derivative dependent stable criteria are obtained and formulated in the form of simple linear matrix inequalities (LMIs). The obtained criteria are dependent on the sizes of delay and its time-derivative and are less conservative than those produced by previous approaches.
\end{abstract}

Keywords

Differential-Algebraic Systems, Stability Analysis, Lyapunov-Krasovskii Functional, Delay Partitioning Approach, Linear Matrix Inequality (LMI)

\section{Introduction}

Differential-algebraic systems, also referred to as singular systems, descriptor systems or generalized state-space systems, arise in a variety of practical systems such as chemical processes, nuclear reactors, biological systems, electrical networks and economy systems. Differential-algebraic systems include not only dynamic equations but also static equations [1] [2]; the study of such systems is much more complicated than that for standard statespace systems. The existence and uniqueness of a solution to a given differential-algebraic system are not always guaranteed and the system can also have undesired impulsive behavior, which can lead to the instability and poor performance [3].

Because of the extensive applications in many practical systems, a great number of fundamental notions and results in control and system theory based on standard state-space systems have been extended successfully to 
differential-algebraic systems. In recent years, much attention has been focused on stability, robust stability and $H_{\infty}$ control problems for differential-algebraic systems, and some results have been derived using the time domain method [4]-[11]. The existing results can be classified into two types: delay-dependent conditions, which include information on the size of delays [12] [13], and delay-independent conditions, which are applicable to delays of arbitrary size [14] [15]. Since the stability of systems depends explicitly on the time-delay, the delay-independent conditions are more conservative, especially for small delays. While the delay-dependent conditions are usually less conservative, and the conservatism will dependent on the chosen of Lyapunov functional, the inequality bounding technique and so on. Two approaches were used to prove the stability of the system in the existing literature. The first approach consists of decomposing the system into fast and slow subsystems and the stability of the slow subsystem is proved using some Lyapunov functional. Then, the fast variables are expressed explicitly by an iterative equation in terms of the slow variables [16]. The second approach consists of constructing a Lyapunov-Krasovskii functional that corresponds directly to the descriptor form of the system [17]. Some other methods have also been provided to reduce the conservative, for example, convex analysis method [18] and delay partitioning approach [19] [20]. To the best of our knowledge, most of the existing delay-dependent asymptotically stable criteria only depend on the upper bound of the delay-derivative, and to the differential-algebraic systems, the delay and its time-derivative dependent stability criterion has not established, which motivates this paper.

This article deals with the problem of asymptotic stability for a class of linear differential-algebraic system with time-varying delay. The obtained criteria depend not only on the upper bound but also on the lower bound of the delay derivative. Based on the Lyapunov functional method and the delay partitioning approach, some delay and its time-derivative dependent stable criteria are obtained. One numerical example is provided to demonstrate the effectiveness of the proposed results. All the developed results are in the LMI framework which makes them more interesting since the solutions are easily obtained using existing powerful tools like the LMI toolbox of Matlab or any equivalent tool.

Notation: Throughout this paper, $A^{\mathrm{T}}$ represents the transpose of $A$; The symbol "*” in matrix inequality denotes the symmetric term of the matrix; $A>0 \quad(<0)$ means $A$ is a symmetrical positive (negative) definite matrix; $\operatorname{Sym}(M)$ stands for $M+M^{\mathrm{T}} ; I$ is a unit matrix.

\section{Problem Statement}

Consider the following differential-algebraic system:

$$
E \dot{x}(t)=A x(t)+A_{1} x(t-\tau(t)) .
$$

where $x(t) \in \mathbf{R}^{n}$ is the state vector, the matrix $E \in \mathbf{R}^{n \times n}$ may be singular, and we assume that $\operatorname{rank}(E)=r \leq n, A$ and $A_{1}$ are constant matrices with appropriate dimensions. $\tau(t)$ is time-varying delay, $\tau(t) \in\left[h_{a}, h_{b}\right], h_{a} \geq 0$, and it is assumed to satisfy $d_{1} \leq \dot{\tau}(t) \leq d_{2}$, where $d_{1}, d_{2}$ are constants. The initial condition is given by $x\left(t_{0}+\theta\right)=\phi(\theta), \theta \in\left[-h_{b}, 0\right], \phi(\theta) \in W$, where $W$ is the space of absolutely continuous function $\phi:\left[-h_{b}, 0\right] \rightarrow \mathbf{R}^{n}$ with the square integrable derivative and with the normal

$$
\|\phi\|_{W}^{2}=|\phi(0)|^{2}+\int_{-h_{b}}^{0}\left[|\phi(s)|^{2}+|\dot{\phi}(s)|^{2}\right] \mathrm{d} s \text {. }
$$

The following definition, lemmas and notation are introduced, which will be used in the proof of the main results.

Definition 1 ([6]) System (1) is said to be regular if the characteristic polynomial, $\operatorname{det}(s E-A)$ is not identically zero.

2) System (1) is said to be impulse-free if $\operatorname{deg}(\operatorname{det}(s E-A))=\operatorname{rank}(E)$.

Lemma 1 ([4]) Let $H, F$ and $G$ be real matrices of appropriate dimensions then, for any scalar $\varepsilon>0$ for all matrices $F$ satisfying $F^{\mathrm{T}} F \leq I$, we have:

$$
H F G+G^{\mathrm{T}} F^{\mathrm{T}} H^{\mathrm{T}} \leq \varepsilon H H^{\mathrm{T}}+\varepsilon^{-1} G^{\mathrm{T}} G .
$$

\section{Main Results}

Theorem 1 System (1) is asymptotically stable for all differentiable delays $\tau(t) \in\left[h_{a}, h_{b}\right]$ with $d_{1} \leq \dot{\tau}(t) \leq d_{2}$, if there exist symmetric positive-definite matrices $Q, R_{i}(i=0,1,2), S_{0}, S_{11}, S_{12}, S_{13}, P^{k}$ satisfying 
$E^{\mathrm{T}} P^{k}=\left(P^{k}\right)^{\mathrm{T}} E \geq 0 \quad(k=1,2,3)$, and appropriately dimensioned matrices $P_{2 j}, P_{3 j}, Y_{1 j}, \quad Y_{2 j}, Z_{1 j}, Z_{2 j}$, $T_{j}(j=1,2), W_{1}$ and $W_{3}$ such that LMIs: $\Psi_{\mid \tau(t)<h_{2}}<0$, for $\tau(t) \rightarrow h_{1}$ and $\tau(t) \rightarrow h_{2}$, and $\Psi_{\mid \tau(t)>h_{2}}<0$, for $\tau(t) \rightarrow h_{2}$ and $\tau(t) \rightarrow h_{3}$, where $\dot{\tau}(t)=d_{1}, d_{2} . \Psi_{\mid \tau(t)<h_{2}}<0$ and $\Psi_{\mid \tau(t)>h_{2}}<0$ are denoted in (8) and (11), respectively.

Proof. The proof of this theorem is divided into two parts. First, we prove that the results, when $\tau(t)<h_{2}$, $\tau(t)>h_{2}$ and $\tau(t)=h_{2}$, respectively, ensure that the derivative of Lyapunov functional is negative. Finally, we prove the results given in the first part ensure that system is regular and impulse-free.

First of all, we divide the delay interval $\left[h_{a}, h_{b}\right]$ into two segments: $\left[h_{1}, h_{2}\right]$ and $\left[h_{2}, h_{3}\right]$, where we denote $h_{1}=h_{a}, h_{3}=h_{b}$, and $h_{2}=\frac{h_{a}+h_{b}}{2}$. Then, system (1) can be represented as

$$
E \dot{x}(t)=A x(t)+\chi_{\left[h_{1}, h_{2}\right]} A_{1} x(t-\tau(t))+\left[1-\chi_{\left[h_{1}, h_{2}\right]}\right] A_{1} x(t-\tau(t))
$$

where $\chi_{\left[h_{1}, h_{2}\right]}: \mathbf{R} \rightarrow\{0,1\}$ is the characteristic function of $\left[h_{1}, h_{2}\right]$

$$
\chi_{\left[h_{1}, h_{2}\right]}(s)= \begin{cases}1, & \text { if } s \in\left[h_{1}, h_{2}\right] \\ 0, & \text { otherwise. }\end{cases}
$$

Consider the following Lyapunov functional:

$$
V\left(x_{t}\right)=V_{1}\left(x_{t}\right)+V_{2}\left(x_{t}\right)+V_{3}\left(x_{t}\right)+V_{4}\left(x_{t}\right)+V_{5}\left(x_{t}\right)
$$

where

$$
\begin{aligned}
& V_{1}\left(x_{t}\right)=x^{\mathrm{T}} E^{\mathrm{T}} P(\tau(t)) x(t), \\
& V_{2}\left(x_{t}\right)=\int_{t-\tau(t)}^{t-h_{1}} x^{\mathrm{T}}(s) Q x(s) \mathrm{d} s, \\
& V_{3}\left(x_{t}\right)=\int_{t-h_{1}}^{t} x^{\mathrm{T}}(s) S_{0} x(s) \mathrm{d} s, \\
& V_{4}\left(x_{t}\right)=\int_{t-h_{2}}^{t-h_{1}} \xi^{\mathrm{T}}(s)\left[\begin{array}{cc}
S_{11} & S_{12} \\
* & S_{13}
\end{array}\right] \xi(s) \mathrm{d} s, \\
& V_{5}\left(x_{t}\right)=\sum_{i=0}^{2}\left(h_{i+1}-h_{i}\right) \int_{-h_{i+1}}^{-h_{i}} \int_{t+\theta}^{t}(E \dot{x}(s))^{\mathrm{T}} R_{i}(E \dot{x}(s)) \mathrm{d} s \mathrm{~d} \theta, \\
& h_{0}=0, \xi(s)=\left[x^{\mathrm{T}}(s), x^{\mathrm{T}}\left(s-\left(h_{2}-h_{1}\right)\right)\right]^{\mathrm{T}}, Q>0, R_{i}>0, S_{0}>0,\left[\begin{array}{cc}
S_{11} & S_{12} \\
* & S_{13}
\end{array}\right]>0 .
\end{aligned}
$$

In addition, we define the continuous function $P(\tau(t))$ as the following form:

$$
\begin{aligned}
P(\tau(t))= & \chi_{\left[h_{1}, h_{2}\right]}(\tau(t))\left[\frac{\tau(t)-h_{1}}{h_{2}-h_{1}} P^{1}+\frac{h_{2}-\tau(t)}{h_{2}-h_{1}} P^{2}\right] \\
& +\left[1-\chi_{\left[h_{1}, h_{2}\right]}(\tau(t))\right]\left[\frac{\tau(t)-h_{2}}{h_{3}-h_{2}} P^{3}+\frac{h_{3}-\tau(t)}{h_{3}-h_{2}} P^{1}\right],
\end{aligned}
$$

When $\tau(t) \neq h_{2}$, one can obtain

$$
\begin{gathered}
\dot{P}(\tau(t))=\dot{\tau}(t)\left[\frac{\chi\left(P^{1}-P^{2}\right)}{h_{2}-h_{1}}+\frac{(1-\chi)\left(P^{3}-P^{1}\right)}{h_{3}-h_{2}}\right], \\
\dot{V}_{1}\left(x_{t}\right)=x^{\mathrm{T}}(t) E^{\mathrm{T}} \dot{P}(\tau(t)) x(t)+2 x^{\mathrm{T}}(t)\left[\chi\left(\frac{\tau(t)-h_{1}}{h_{2}-h_{1}} P^{1}+\frac{h_{2}-\tau(t)}{h_{2}-h_{1}} P^{2}\right)\right. \\
\left.+(1-\chi)\left(\frac{\tau(t)-h_{2}}{h_{3}-h_{2}} P^{3}+\frac{h_{3}-\tau(t)}{h_{3}-h_{2}} P^{1}\right)\right] E \dot{x}(t),
\end{gathered}
$$




$$
\dot{V}_{5}\left(x_{t}\right)=(E \dot{x}(t))^{\mathrm{T}}\left[\sum_{i=0}^{2}\left(h_{i+1}-h_{i}\right)^{2} R_{i}\right](E \dot{x}(t))-\sum_{i=0}^{2}\left(h_{i+1}-h_{i}\right) \int_{t-h_{i+1}}^{t-h_{i}}(E \dot{x}(s))^{\mathrm{T}} R_{i}(E \dot{x}(s)) \mathrm{d} s
$$

To obtain the main results, we consider the following three cases: $\tau(t) \in\left[h_{1}, h_{2}\right), \tau(t) \in\left(h_{2}, h_{3}\right]$ and $\tau(t)=h_{2}$.

Case 1: $\tau(t) \in\left[h_{1}, h_{2}\right)$

Using the fact that $\int_{t-h_{j+1}}^{t-h_{j}} f_{j}(s) \mathrm{d} s=\int_{t-h_{j+1}}^{t-\tau(t)} f_{j}(s) \mathrm{d} s+\int_{t-\tau(t)}^{t-h_{j}} f_{j}(s) \mathrm{d} s$, where $f_{j}(s)=(E \dot{x}(s))^{\mathrm{T}} R_{i}(E \dot{x}(s))$, we easily obtain the following inequalities by Jensen's inequality:

$$
\begin{gathered}
\int_{t-h_{i+1}}^{t-h_{i}}\left(h_{i+1}-h_{i}\right) f_{i}(s) \mathrm{d} s \geq \int_{t-h_{i+1}}^{t-h_{i}}(E \dot{x}(s))^{\mathrm{T}} \mathrm{d} s R_{i} \int_{t-h_{i+1}}^{t-h_{i}}(E \dot{x}(s)) \mathrm{d} s, \\
\int_{t-\tau(t)}^{t-h_{j}}\left(h_{j+1}-h_{j}\right) f_{j}(s) \mathrm{d} s \geq\left(\tau(t)-h_{j}\right)\left(h_{j+1}-h_{j}\right) v_{j 1}^{\mathrm{T}} R_{j} v_{j 1}, \\
\int_{t-h_{j+1}}^{t-\tau(t)}\left(h_{j+1}-h_{j}\right) f_{j}(s) \mathrm{d} s \geq\left(h_{j+1}-\tau(t)\right)\left(h_{j+1}-h_{j}\right) v_{j 2}^{\mathrm{T}} R_{j} v_{j 2},
\end{gathered}
$$

where $v_{j 1}=\frac{1}{\tau(t)-h_{j}} \int_{t-\tau(t)}^{t-h_{j}}(E \dot{x}(s)) \mathrm{d} s, \quad v_{j 2}=\frac{1}{h_{j+1}-\tau(t)} \int_{t-h_{j+1}}^{t-\tau(t)}(E \dot{x}(s)) \mathrm{d} s, \quad i=0,2, \quad j=1$. Then, the timederivative of $V\left(x_{t}\right)$ along the solution of (2) is given by

$$
\begin{aligned}
\dot{V}\left(x_{t}\right)_{\mid \tau(t)<h_{2}}= & \dot{V}_{1}\left(x_{t}\right)_{\mid \tau(t)<h_{2}}+(E \dot{x}(t))^{\mathrm{T}}\left[\sum_{i=0}^{2}\left(h_{i+1}-h_{i}\right)^{2} R_{i}\right](E \dot{x}(t)) \\
& +x^{\mathrm{T}}\left(t-h_{1}\right) Q x\left(t-h_{1}\right)-(1-\dot{\tau}(t)) x^{\mathrm{T}}(t-\tau(t)) Q x(t-\tau(t)) \\
& +x^{\mathrm{T}}(t) S_{0} x(t)-x^{\mathrm{T}}\left(t-h_{1}\right) S_{0} x\left(t-h_{1}\right) \\
& +\left[\begin{array}{c}
x\left(t-h_{1}\right) \\
x\left(t-h_{2}\right)
\end{array}\right]^{\mathrm{T}}\left[\begin{array}{ll}
S_{11} & S_{12} \\
* & S_{13}
\end{array}\right]\left[\begin{array}{l}
x\left(t-h_{1}\right) \\
x\left(t-h_{2}\right)
\end{array}\right] \\
& -\left[\begin{array}{l}
x\left(t-h_{2}\right) \\
x\left(t-h_{3}\right)
\end{array}\right]^{\mathrm{T}}\left[\begin{array}{ll}
S_{11} & S_{12} \\
* & S_{13}
\end{array}\right]\left[\begin{array}{l}
x\left(t-h_{2}\right) \\
x\left(t-h_{3}\right)
\end{array}\right] \\
& -\left[x(t)-x\left(t-h_{1}\right)\right]^{\mathrm{T}} E^{\mathrm{T}} R_{0} E\left[x(t)-x\left(t-h_{1}\right)\right] \\
& -\left[x\left(t-h_{2}\right)-x\left(t-h_{3}\right)\right]^{\mathrm{T}} E^{\mathrm{T}} R_{2} E\left[x\left(t-h_{2}\right)-x\left(t-h_{3}\right)\right] \\
& -\left(\tau(t)-h_{j}\right)\left(h_{j+1}-h_{j}\right) v_{j 1}^{\mathrm{T}} R_{j} v_{j 1} \\
& -\left(h_{j+1}-\tau(t)\right)\left(h_{j+1}-h_{j}\right) v_{j 2}^{\mathrm{T}} R_{j} v_{j 2},
\end{aligned}
$$

Letting $\eta_{1}(t)=\left[x^{\mathrm{T}}(t), E \dot{x}^{\mathrm{T}}(t), x^{\mathrm{T}}\left(t-h_{1}\right), x^{\mathrm{T}}\left(t-h_{2}\right), v_{11}^{\mathrm{T}}, v_{12}^{\mathrm{T}}, x^{\mathrm{T}}(t-\tau(t)), x^{\mathrm{T}}\left(t-h_{3}\right)\right]^{\mathrm{T}}$, and adding the following terms

$$
\begin{aligned}
0 & =2\left[x^{\mathrm{T}}(t) Y_{11}^{\mathrm{T}}+\dot{x}^{\mathrm{T}}(t) E^{\mathrm{T}} Y_{21}^{\mathrm{T}}+x^{\mathrm{T}}(t-\tau(t)) T_{1}^{\mathrm{T}}\right]\left[-E x\left(t-h_{1}\right)+E x(t-\tau(t))+\left(\tau(t)-h_{1}\right) v_{11}\right], \\
0 & =2\left[x^{\mathrm{T}}(t) Z_{11}^{\mathrm{T}}+\dot{x}^{\mathrm{T}}(t) E^{\mathrm{T}} Z_{21}^{\mathrm{T}}+x^{\mathrm{T}}\left(t-h_{3}\right) W_{3}^{\mathrm{T}}\right]\left[E x\left(t-h_{2}\right)-E x(t-\tau(t))+\left(h_{2}-\tau(t)\right) v_{12}\right], \\
0 & =2\left[x^{\mathrm{T}}(t) P_{21}^{\mathrm{T}}+\dot{x}^{\mathrm{T}}(t) E^{\mathrm{T}} P_{31}^{\mathrm{T}}\right]\left[A x(t)+A_{1} x(t-\tau(t))-E \dot{x}(t)\right]
\end{aligned}
$$

to (5) gives

$$
V\left(x_{t}\right)_{\mid \tau(t)<h_{2}} \leq \eta_{1}^{\mathrm{T}}(t) \Psi_{\mid \tau(t)<h_{2}} \eta_{1}(t) \leq-\alpha_{1}|x(t)|^{2}
$$

for some scalar $\alpha_{1}>0$, if 


$$
\Psi_{\mid \tau(t)<h_{2}}=\left[\begin{array}{cccccccc}
\Pi_{11} & \Pi_{12} & \Pi_{13} & Z_{11}^{\mathrm{T}} E & \Pi_{15} & \Pi_{16} & \Pi_{17} & 0 \\
* & \Pi_{22} & -Y_{21}^{\mathrm{T}} E & Z_{21}^{\mathrm{T}} E & \Pi_{25} & \Pi_{26} & \Pi_{27} & 0 \\
* & * & \Pi_{33} & S_{12} & 0 & 0 & -E^{\mathrm{T}} T_{1} & 0 \\
* & * & * & \Pi_{44} & 0 & 0 & 0 & \Pi_{48} \\
* & * & * & * & \Pi_{55} & 0 & \Pi_{57} & 0 \\
* & * & * & * & * & \Pi_{66} & 0 & \Pi_{68} \\
* & * & * & * & * & * & \Pi_{77} & -E^{\mathrm{T}} W_{3} \\
* & * & * & * & * & * & * & \Pi_{88}
\end{array}\right]<0
$$

where

$$
\begin{aligned}
\Pi_{11}= & \frac{\dot{\tau}(t)}{h_{2}-h_{1}} E^{\mathrm{T}}\left(P^{1}-P^{2}\right)+\frac{\tau(t)-h_{1}}{h_{2}-h_{1}} \operatorname{Sym}\left(\left(P^{1}\right)^{\mathrm{T}} A\right) \\
& +\frac{h_{2}-\tau(t)}{h_{2}-h_{1}} \operatorname{Sym}\left(\left(P^{2}\right)^{\mathrm{T}} A\right)+S_{0}-E^{\mathrm{T}} R_{0} E+A^{\mathrm{T}} P_{21}+P_{21}^{\mathrm{T}} A, \\
\Pi_{12}= & -P_{21}^{\mathrm{T}}+A^{\mathrm{T}} P_{31}, \quad \Pi_{13}=E^{\mathrm{T}} R_{0} E-Y_{11} E, \\
\Pi_{15}= & \left(\tau(t)-h_{1}\right) Y_{11}^{\mathrm{T}}, \quad \Pi_{16}=\left(h_{2}-\tau(t)\right) Z_{11}^{\mathrm{T}}, \\
\Pi_{17}= & \frac{\tau(t)-h_{1}}{h_{2}-h_{1}} P^{1} A_{1}+\frac{h_{2}-\tau(t)}{h_{2}-h_{1}} P^{2} A_{1}+Y_{11}^{\mathrm{T}} E-Z_{11}^{\mathrm{T}} E+P_{21}^{\mathrm{T}} A_{1}, \\
\Pi_{22}= & -P_{31}^{\mathrm{T}}-P_{31}+\sum_{i=0}^{2}\left(h_{i+1}-h_{i}\right)^{2} R_{i}, \quad \Pi_{25}=\left(\tau(t)-h_{1}\right) Y_{21}^{\mathrm{T}}, \\
\Pi_{26}= & \left(h_{2}-\tau(t)\right) Z_{21}^{\mathrm{T}}, \quad \Pi_{27}=Y_{21}^{\mathrm{T}} E-Z_{21}^{\mathrm{T}} E+P_{31}^{\mathrm{T}} A_{1}, \\
\Pi_{33}= & Q-S_{0}+S_{11}-E^{\mathrm{T}} R_{0} E, \quad \Pi_{44}=-S_{11}+S_{13}-E^{\mathrm{T}} R_{2} E, \\
\Pi_{48}= & -S_{12}+E^{\mathrm{T}} R_{2} E+E^{\mathrm{T}} W_{3}, \quad \Pi_{55}=-\left(\tau(t)-h_{1}\right)\left(h_{2}-h_{1}\right) R_{1}, \\
\Pi_{57}= & \left(\tau(t)-h_{1}\right) T_{1}, \quad \Pi_{66}=-\left(h_{2}-\tau(t)\right)\left(h_{2}-h_{1}\right) R_{1}, \\
\Pi_{68}= & \left(h_{2}-\tau(t)\right) W_{3}, \quad \Pi_{77}=-(1-\dot{\tau}(t)) Q+T_{1}^{\mathrm{T}} E+E^{\mathrm{T}} T_{1}, \\
\Pi_{88}= & -S_{13}-E^{\mathrm{T}} R_{2} E .
\end{aligned}
$$

Inequality (8) contains two variables which make it difficult to solve by LMI tool. In order to overcome this difficulty, we seek the sufficient conditions for inequality (8). When $\tau(t) \rightarrow h_{1}$ and $\tau(t) \rightarrow h_{2}$, the inequality (8) leads to the following LMIs:

$$
\Psi_{1}=\left[\begin{array}{ccccccc}
\bar{\Pi}_{11} & \Pi_{12} & \Pi_{13} & Z_{11}^{\mathrm{T}} E & \left(h_{2}-h_{1}\right) Z_{11}^{\mathrm{T}} & \bar{\Pi}_{17} & 0 \\
* & \Pi_{22} & -Y_{21}^{\mathrm{T}} E & Z_{21}^{\mathrm{T}} E & \left(h_{2}-h_{1}\right) Z_{21}^{\mathrm{T}} & \Pi_{27} & 0 \\
* & * & \Pi_{33} & S_{12} & 0 & -E^{\mathrm{T}} T_{1} & 0 \\
* & * & * & \Pi_{44} & 0 & 0 & \Pi_{48} \\
* & * & * & * & -\left(h_{2}-h_{1}\right)^{2} R_{1} & 0 & \left(h_{2}-h_{1}\right) W_{3} \\
* & * & * & * & * & \Pi_{77} & -E^{\mathrm{T}} W_{3} \\
* & * & * & * & * & * & \Pi_{88}
\end{array}\right]<0
$$

and 


$$
\Psi_{2}=\left[\begin{array}{ccccccc}
\hat{\Pi}_{11} & \Pi_{12} & \Pi_{13} & Z_{11}^{\mathrm{T}} E & \left(h_{2}-h_{1}\right) Y_{11}^{\mathrm{T}} & \hat{\Pi}_{17} & 0 \\
* & \Pi_{22} & -Y_{21}^{\mathrm{T}} E & Z_{21}^{\mathrm{T}} E & \left(h_{2}-h_{1}\right) Y_{21}^{\mathrm{T}} & \Pi_{27} & 0 \\
* & * & \Pi_{33} & S_{12} & 0 & -E^{\mathrm{T}} T_{1} & 0 \\
* & * & * & \Pi_{44} & 0 & 0 & \Pi_{48} \\
* & * & * & * & -\left(h_{2}-h_{1}\right)^{2} R_{1} & \left(h_{2}-h_{1}\right) T_{1} & 0 \\
* & * & * & * & * & \Pi_{77} & -E^{\mathrm{T}} W_{3} \\
* & * & * & * & * & * & \Pi_{88}
\end{array}\right]<0
$$

where

$$
\bar{\Pi}_{11}=\Pi_{11 \tau \tau(t)=h_{1}}, \quad \bar{\Pi}_{17}=\Pi_{17 \mid \tau(t)=h_{1}}, \quad \hat{\Pi}_{11}=\Pi_{11 \mid \tau(t)=h_{2}}, \quad \hat{\Pi}_{17}=\Pi_{17 \mid \tau(t)=h_{2}} .
$$

Note that we have omitted the zero row and zero column in $\Psi_{1}$ and $\Psi_{2}$. Letting $\eta_{1 i}=\left[x^{\mathrm{T}}(t),(E \dot{x}(t))^{\mathrm{T}}, x^{\mathrm{T}}\left(t-h_{1}\right), x^{\mathrm{T}}\left(t-h_{2}\right), v_{j i}^{\mathrm{T}}, x^{\mathrm{T}}(t-\tau(t)), x^{\mathrm{T}}\left(t-h_{3}\right)\right]^{\mathrm{T}}$, the latter two LMIs imply (8), which is because

$$
\begin{aligned}
& \frac{\tau(t)-h_{1}}{h_{2}-h_{1}} \eta_{11}^{\mathrm{T}}(t) \Psi_{2} \eta_{11}(t)+\frac{h_{2}-\tau(t)}{h_{2}-h_{1}} \eta_{12}^{\mathrm{T}}(t) \Psi_{1} \eta_{12}(t) \\
& =\eta_{1}^{\mathrm{T}}(t) \Psi_{\mid \tau(t)<h_{2}} \eta_{1}(t) \leq-\alpha_{1}|x(t)|^{2} .
\end{aligned}
$$

Thus, $\Psi_{\mid \tau(t)<h_{2}}$ is convex in $\tau(t) \in\left[h_{1}, h_{2}\right)$.

When $\dot{\tau}(t)=d_{i}$, it follows from (9) that

$$
\Psi_{1 i}=\Psi_{1 \mid i(t)=d_{i}}<0, \quad i=1,2 .
$$

The LMI (11) implies (9), since

$$
\frac{d_{2}-\dot{\tau}(t)}{d_{2}-d_{1}} \Psi_{11}+\frac{\dot{\tau}(t)-d_{2}}{d_{2}-d_{1}} \Psi_{12}=\Psi_{1}<0 .
$$

Thus, $\Psi_{1}$ is convex in $\dot{\tau}(t) \in\left[d_{1}, d_{2}\right]$. Similarly, we can obtain that $\Psi_{2}$ is also convex in $\dot{\tau}(t) \in\left[d_{1}, d_{2}\right]$. Case 2: $\tau(t) \in\left(h_{2}, h_{3}\right]$

By the definition of the characteristic function $\chi=0$, we apply the above arguments and representations with $i=0,1$ and $j=2$. In addition, we replace (6) with the following equations:

$$
\begin{aligned}
& 0=2 {\left[x^{\mathrm{T}}(t) Y_{12}^{\mathrm{T}}+\dot{x}^{\mathrm{T}}(t) E^{\mathrm{T}} Y_{22}^{\mathrm{T}}+x^{\mathrm{T}}(t-\tau(t)) T_{2}^{\mathrm{T}}+x^{\mathrm{T}}\left(t-h_{1}\right) W_{1}^{\mathrm{T}}\right] } \\
& \cdot\left[-E x\left(t-h_{2}\right)+E x(t-\tau(t))+\left(\tau(t)-h_{2}\right) v_{11}\right], \\
& 0=2 {\left[x^{\mathrm{T}}(t) Z_{12}^{\mathrm{T}}+\dot{x}^{\mathrm{T}}(t) E^{\mathrm{T}} Z_{22}^{\mathrm{T}}\right]\left[E x\left(t-h_{3}\right)-E x(t-\tau(t))+\left(h_{3}-\tau(t)\right) v_{22}\right], } \\
& 0=2\left[x^{\mathrm{T}}(t) P_{22}^{\mathrm{T}}+\dot{x}^{\mathrm{T}}(t) E^{\mathrm{T}} P_{32}^{\mathrm{T}}\right]\left[A x(t)+A_{1} x(t-\tau(t))-E \dot{x}(t)\right],
\end{aligned}
$$

and letting $\eta_{2}(t)=\left[x^{\mathrm{T}}(t),(E \dot{x}(t))^{\mathrm{T}}, x^{\mathrm{T}}\left(t-h_{1}\right), x^{\mathrm{T}}\left(t-h_{2}\right), v_{21}^{\mathrm{T}}, v_{22}^{\mathrm{T}}, x^{\mathrm{T}}(t-\tau(t)), x^{\mathrm{T}}\left(t-h_{3}\right)\right]^{\mathrm{T}}$. It is easy to obtain that

$$
\dot{V}\left(x_{t}\right)_{\mid \tau(t)>h_{2}} \leq \eta_{2}^{\mathrm{T}}(t) \Psi_{\mid \tau(t)>h_{2}} \eta_{2}(t) \leq-\alpha_{2}|x(t)|^{2},
$$

for some scala $\alpha_{2}>0$, where 


$$
\Psi_{\mid r(t)>h_{2}}=\left[\begin{array}{cccccccc}
\tilde{\Pi}_{11} & \tilde{\Pi}_{12} & E^{\mathrm{T}} R_{0} E & -Y_{12}^{\mathrm{T}} E & \tilde{\Pi}_{15} & \tilde{\Pi}_{16} & \tilde{\Pi}_{17} & Z_{12}^{\mathrm{T}} E \\
* & \tilde{\Pi}_{22} & 0 & -Y_{22}^{\mathrm{T}} E & \tilde{\Pi}_{25} & \tilde{\Pi}_{26} & \tilde{\Pi}_{27} & Z_{22}^{\mathrm{T}} E \\
* & * & \tilde{\Pi}_{33} & \tilde{\Pi}_{34} & \tilde{\Pi}_{35} & 0 & W_{1}^{\mathrm{T}} E & 0 \\
* & * & * & \tilde{\Pi}_{44} & 0 & 0 & -E^{\mathrm{T}} T_{2} & -S_{12} \\
* & * & * & * & \tilde{\Pi}_{55} & 0 & \tilde{\Pi}_{57} & 0 \\
* & * & * & * & * & \tilde{\Pi}_{66} & 0 & 0 \\
* & * & * & * & * & * & \tilde{\Pi}_{77} & 0 \\
* & * & * & * & * & * & * & -S_{13}
\end{array}\right]<0
$$

where

$$
\begin{aligned}
\tilde{\Pi}_{11}= & \frac{\dot{\tau}(t)}{h_{3}-h_{2}} E^{\mathrm{T}}\left(P^{3}-P^{1}\right)+\frac{\tau(t)-h_{2}}{h_{3}-h_{2}} \operatorname{Sym}\left(\left(P^{3}\right)^{\mathrm{T}} A\right) \\
& +\frac{h_{3}-\tau(t)}{h_{3}-h_{2}} \operatorname{Sym}\left(\left(P^{1}\right)^{\mathrm{T}} A\right)+S_{0}-E^{\mathrm{T}} R_{0} E+A^{\mathrm{T}} P_{22}+P_{22}^{\mathrm{T}} A, \\
\tilde{\Pi}_{12}= & -P_{22}^{\mathrm{T}}+A^{\mathrm{T}} P_{32}, \quad \tilde{\Pi}_{15}=\left(\tau(t)-h_{2}\right) Y_{12}^{\mathrm{T}}, \quad \tilde{\Pi}_{16}=\left(h_{3}-\tau(t)\right) Z_{12}^{\mathrm{T}}, \\
\tilde{\Pi}_{17}= & \frac{\tau(t)-h_{2}}{h_{3}-h_{2}}\left(P^{3}\right)^{\mathrm{T}} A_{1}+\frac{h_{3}-\tau(t)}{h_{3}-h_{2}}\left(P^{1}\right)^{\mathrm{T}} A_{1}+Y_{12}^{\mathrm{T}} E-Z_{12}^{\mathrm{T}} E+P_{22}^{\mathrm{T}} A_{1}, \\
\tilde{\Pi}_{22}= & -P_{32}^{\mathrm{T}}-P_{32}+\sum_{i=0}^{2}\left(h_{i+1}-h_{i}\right)^{2} R_{i}, \quad \tilde{\Pi}_{25}=\left(\tau(t)-h_{2}\right) Y_{22}^{\mathrm{T}}, \\
\tilde{\Pi}_{26}= & \left(h_{3}-\tau(t)\right) Z_{22}^{\mathrm{T}}, \quad \tilde{\Pi}_{27}=Y_{22}^{\mathrm{T}} E-Z_{22}^{\mathrm{T}} E+P_{32}^{\mathrm{T}} A_{1}, \\
\tilde{\Pi}_{33}= & Q-S_{0}+S_{11}-E^{\mathrm{T}}\left(R_{0}+R_{1}\right) E, \quad \tilde{\Pi}_{34}=E^{\mathrm{T}} R_{1} E-W_{1}^{\mathrm{T}} E+S_{12}, \\
\tilde{\Pi}_{35}= & \left(\tau(t)-h_{2}\right) W_{1}^{\mathrm{T}}, \quad \tilde{\Pi}_{44}=-S_{11}+S_{13}-E^{\mathrm{T}} R_{1} E, \\
\tilde{\Pi}_{55}= & -\left(\tau(t)-h_{2}\right)\left(h_{3}-h_{2}\right) R_{2}, \quad \tilde{\Pi}_{57}=\left(\tau(t)-h_{2}\right) T_{2}, \\
\tilde{\Pi}_{66}= & -\left(h_{3}-\tau(t)\right)\left(h_{3}-h_{2}\right) R_{2}, \quad \tilde{\Pi}_{77}=-(1-\dot{\tau}(t)) Q+T_{2}^{\mathrm{T}} E+E^{\mathrm{T}} T_{2} .
\end{aligned}
$$

Similar to the case I, we obtain the results when $\tau(t) \rightarrow h_{2}$ and $\tau(t) \rightarrow h_{3}$, which can be marked as $\bar{\Psi}_{1}<0$ and $\bar{\Psi}_{2}<0$, respectively. Further, we can verify $\Psi_{\mid \tau(t)>h_{2}}$ is convex in $\tau(t) \in\left(h_{2}, h_{3}\right], \bar{\Psi}_{1}$ and $\bar{\Psi}_{2}$ are also convex in $\dot{\tau}(t) \in\left[d_{1}, d_{2}\right]$.

From Case 1 and Case 2, we have

$$
\dot{V}_{\mid \tau(t) \neq h_{2}} \leq \chi_{\left[h_{1}, h_{2}\right]}(\tau(t)) \eta_{1}^{\mathrm{T}}(t) \Psi_{\mid \tau(t)<h_{2}} \eta_{1}(t)+\left(1-\chi_{\left[h_{1}, h_{2}\right]}(\tau(t))\right) \eta_{2}^{\mathrm{T}}(t) \Psi_{\mid \tau(t)>h_{2}} \eta_{2}(t) \leq-\alpha|x(t)|^{2},
$$

for some scalar $\alpha>0$.

Case 3: $\tau(t)=h_{2}$

$$
\dot{V}_{\mid \tau(t)=h_{2}} \leq \max \left\{\eta_{1}^{\mathrm{T}}(t) \Psi_{\mid \tau(t)<h_{2}} \eta_{1}(t), \eta_{2}^{\mathrm{T}}(t) \Psi_{\mid \tau(t)>h_{2}} \eta_{2}(t)\right\} \leq-\alpha|x(t)|^{2} .
$$

Therefore, $\dot{V}\left(x_{t}\right) \leq-\alpha|x(t)|^{2}$ when $\tau(t) \in\left[h_{a}, h_{b}\right], \quad \dot{\tau}(t) \in\left[d_{1}, d_{2}\right]$.

Now the asymptotic stability of system (1) can not be obtained yet, since the existence and uniqueness of a solution to system (1) are not always guaranteed and the system may have undesired impulsive behavior. In the following, we will prove that the above-mentioned results ensure the regular and impulse-free. It follows form (8), that 


$$
\left[\begin{array}{cc}
\Pi_{11} & \Pi_{12} \\
* & \Pi_{22}
\end{array}\right]-\left[\begin{array}{cc}
S_{0} & 0 \\
* & \sum_{i=0}^{2}\left(h_{i+1}-h_{i}\right)^{2} R_{i}
\end{array}\right]<0 .
$$

Pre- and post-multiplying (15) by $\left[I, A^{\mathrm{T}}\right]$ and $\left[I, A^{\mathrm{T}}\right]^{\mathrm{T}}$, respectively, we obtain

$$
\frac{\dot{\tau}(t)}{h_{2}-h_{1}} E^{\mathrm{T}}\left(P^{1}-P^{2}\right)+\frac{\tau(t)-h_{1}}{h_{2}-h_{1}} \operatorname{Sym}\left(\left(P^{1}\right)^{\mathrm{T}} A\right)+\frac{h_{2}-\tau(t)}{h_{2}-h_{1}} \operatorname{Sym}\left(\left(P^{2}\right)^{\mathrm{T}} A\right)-E^{\mathrm{T}} R_{0} E<0
$$

Since $\operatorname{rank} E=r \leq n$, there must exist two invertible matrices $G$ and $H \in \mathbb{R}^{n \times n}$ such that

$$
\hat{E}=G E H=\left[\begin{array}{cc}
I_{r} & 0 \\
0 & 0
\end{array}\right] .
$$

Similar to (17), we define

$$
\hat{A}=G A H=\left[\begin{array}{ll}
A_{11} & A_{12} \\
A_{21} & A_{22}
\end{array}\right], \quad \hat{P}^{i}=G^{-\mathrm{T}} P^{i} H=\left[\begin{array}{ll}
P_{1}^{i} & P_{2}^{i} \\
P_{3}^{i} & P_{4}^{i}
\end{array}\right], i=1,2 .
$$

Then, using $E^{\mathrm{T}} P^{k}=\left(P^{k}\right)^{\mathrm{T}} E \geq 0$, it can be shown that $P_{2}^{i}=0$. Pre- and post-multiplying (16) by $H^{\mathrm{T}}$ and $H$, we can formulate the following inequality easily

$$
\left[\begin{array}{cc}
\star & \star \\
\star & \operatorname{Sym}\left(A_{22}^{\mathrm{T}}\left(P_{4}^{1}+P_{4}^{2}\right)\right)
\end{array}\right]<0
$$

where $\star$ will be irrelevant to the following discussion. Form (18) we get $\operatorname{Sym}\left(A_{22}^{\mathrm{T}}\left(P_{4}^{1}+P_{4}^{2}\right)\right)<0$, and thus $A_{22}$ is nonsingular, which implies that $\operatorname{det}(s E-A)$ is not identically zero and $\operatorname{deg}(\operatorname{det}(s E-A))=r=\operatorname{rank} E$. Hence, by Definition 1, the above-mentioned results guarantee system (1) is regular and impulse-free. This completes the proof.

When the matrix $E$ is nonsingular, the result in this case can be obtained by setting $E$ equal to $I$ with the appropriate transformations. The corresponding result is given by the following corollary:

Corollary 1 System (1) with $E=I$ is asymptotically stable for all differentiable delays $\tau(t) \in\left[h_{a}, h_{b}\right]$ with $d_{1} \leq \dot{\tau}(t) \leq d_{2}$, if there exist symmetric positive-definite matrices $Q, R_{i}(i=0,1,2), S_{0}, S_{11}, S_{12}, S_{13}, P^{k}$ $(k=1,2,3)$, and appropriately dimensioned matrices $P_{2 j}, P_{3 j}, Y_{1 j}, Y_{2 j}, Z_{1 j}, Z_{2 j}, T_{j}(j=1,2), W_{1}$ and $W_{3}$ such that LMIs: $\Psi_{\mid \tau(t)<h_{2}}<0$, for $\tau(t) \rightarrow h_{1}$ and $\tau(t) \rightarrow h_{2}$, and $\Psi_{\mid \tau(t)>h_{2}}<0$, for $\tau(t) \rightarrow h_{2}$ and $\tau(t) \rightarrow h_{3}$, where $\dot{\tau}(t)=d_{1}, d_{2} . \Psi_{\mid \tau(t)<h_{2}}<0$ and $\Psi_{\mid \tau(t)>h_{2}}<0$ are denoted in (8) and (11), respectively.

When $d_{1}$ is unknown, let $P=P^{k} \quad(k=1,2,3)$ and $\dot{\tau}(t)=d_{2}$, we have the following corollary.

Corollary 2 System (1) is asymptotically stable for all differentiable delays $\tau(t) \in\left[h_{a}, h_{b}\right]$ with $i(t) \leq d_{2}$, if there exist symmetric positive-definite matrices $Q, R_{i}(i=0,1,2), S_{0}, S_{11}, S_{12}, S_{13}, P$ satisfying $E^{\mathrm{T}} P=P^{\mathrm{T}} E \geq 0$, and appropriately dimensioned matrices $P_{2 j}, P_{3 j}, Y_{1 j}, Y_{2 j}, Z_{1 j}, Z_{2 j}, T_{j}(j=1,2), W_{1}$ and $W_{3}$ such that LMIs: $\Psi_{\mid \tau(t)<h_{2}}<0$, for $\tau(t) \rightarrow h_{1}$ and $\tau(t) \rightarrow h_{2}$, and $\Psi_{\mid \tau(t)>h_{2}}<0$, for $\tau(t) \rightarrow h_{2}$ and $\tau(t) \rightarrow h_{3}$, where $\dot{\tau}(t)=d_{2} . \quad \Psi_{\mid \tau(t)<h_{2}}<0$ and $\Psi_{\mid \tau(t)>h_{2}}<0$ are denoted in (8) and (11), respectively.

Remark 1. It should be pointed out that if $h_{a}$ is big enough, delay partitioning of $\left[0, h_{a}\right]$ may improve the results. In addition, the better results may be obtained if we divide the delay interval $\left[h_{a}, h_{b}\right]$ into $N(N>2)$ segments.

\section{A Numerical Example}

In this section, a numerical example will be presented to show the validity of the main results derived above.

Example Consider the following linear differential-algebraic system described by systems (1) with

$$
E=\left[\begin{array}{ll}
1 & 0 \\
0 & 0
\end{array}\right], \quad A=\left[\begin{array}{cc}
-0.3 & 0.5 \\
-0.3 & -1
\end{array}\right], \quad A_{1}=\left[\begin{array}{cc}
-0.5 & 0 \\
0 & -0.1
\end{array}\right] .
$$


For $h_{a}=0$, choosing $d_{1}=-0.3$ and $d_{2}=0.9$ and applying Theorem 1 , the maximum values of $h_{b}$ is 5.27 , which guarantees that the system is asymptotically stable.

\section{Conclusion}

In this paper, the asymptotic stability of differential-algebraic system with time-varying delay has been investigated. Some delay and its time-derivative dependent asymptotically stable criteria have been obtained by decomposing time-varying delay in a convex set. The obtained criteria depend not only on the upper but also on the lower bound of the delay derivative. One numerical example has been given to illustrate the effectiveness of the proposed main results.

\section{Acknowledgements}

We thank the Editor and the referee for their comments. This work was supported by A Project Supported by Scientific Research Fund of Sichuan Provincial Education Department (16ZA0146) and the Doctoral Research Foundation of Southwest University of Science and Technology (13zx7141).

\section{References}

[1] Lin, C., Wang, Q.G. and Lee, T.H. (2005) Robust Normalization and Stabilization of Uncertain Descriptor Systems with Norm-Bounded Perturbations. IEEE Transactions on Automatic Control, 50, 515-520. http://dx.doi.org/10.1109/TAC.2005.844908

[2] Xu, S.Y. and Lam, J. (2006) Robust Control and Filtering of Singular Systems. Springer-Verlag, Berlin.

[3] Dai, L. (1989) Singular Control Systems, Lecture Notes in Control and Information Sciences. Springer-Verlag, New York.

[4] Boukas, E.K. (2009) Delay-Dependent Robust Stabilizability of Singular Linear Systems with Delays. Stochastic Analysis and Applications, 27, 637-655. http://dx.doi.org/10.1080/07362990902976165

[5] Chou, J.H., Chen, S.H. and Zhang, Q.L. (2006) Robust Controllability for Linear Uncertain Descriptor Systems. Linear Algebra and its Applications, 414, 632-651. http://dx.doi.org/10.1016/j.laa.2005.11.005

[6] Haidar, A. and Boukas, E.K. (2009) Exponential Stability of Singular Systems with Multiple Time-Varying Delays. Automatica, 45, 539-545. http://dx.doi.org/10.1016/j.automatica.2008.08.019

[7] Lu, R.Q., Xu, Y. and Xue, A.K. (2010) $H_{\infty}$ filtering for Singular Systems with Communication Delays. Signal Process, 90, 1240-1248. http://dx.doi.org/10.1016/j.sigpro.2009.10.007

[8] Virnik, E. (2008) Stability Analysis of Positive Descriptor Systems. Linear Algebra and Its Applications, 429, 26402659. http://dx.doi.org/10.1016/j.laa.2008.03.002

[9] Wo, S.L., Zou, Y., Chen, Q.W. and Xu, S.Y. (2009) Non-Fragile Controller Design for Discrete Descriptor Systems. Journal of The Franklin Institute, 346, 914-922. http://dx.doi.org/10.1016/j.jfranklin.2009.07.008

[10] Long, S.H. and Zhong, S.M. (2016) $H_{\infty}$ Control for a Class of Discrete-Time Singular Systems via Dynamic Feedback Controller. Applied Mathematics Letters, 58, 110-118. http://dx.doi.org/10.1016/j.aml.2016.02.004

[11] Niamsup, P. and Phat, V.N. (2016) A New Result on Finite-Time Control of Singular Linear Time-Delay Systems. Applied Mathematics Letters, 60, 1-7. http://dx.doi.org/10.1016/j.aml.2016.03.015

[12] Deng, S.J., Liao, X.F. and Guo, S.T. (2009) Asymptotic Stability Analysis of Certain Neutral Differential Equations: A Descriptor System Approach. Mathematics and Computers in Simulation, 79, 2981-2993. http://dx.doi.org/10.1016/j.matcom.2009.01.005

[13] Qian, W., Liu, J., Sun, Y.X. and Fei, S.M. (2010) A Less Conservative Robust Stability Criteria for Uncertain Neutral Systems with Mixed Delays. Mathematics and Computers in Simulation, 80, 1007-1017. http://dx.doi.org/10.1016/j.matcom.2009.12.007

[14] Xu, S.Y., Lam, J. and Yang, C. (2002) Robust $H_{\infty}$ Control for Discrete Singular Systems with State Delay and Parameter Uncertainty. Dynamics of Continuous, Discrete and Impulsive Systems, 9, 539-554.

[15] Yang, C.Y., Zhang, Q.L. and Zhou, L.N. (2007) Strongly Absolute Stability of Lur'e Type Differential-Algebraic Systems. Journal of Mathematical Analysis and Applications, 336, 188-204. http://dx.doi.org/10.1016/j.jmaa.2007.02.067

[16] Xu, S.Y., Van Dooren, P., Stefan, R. and Lam, J. (2002) Robust Stability and Stabilization for Singular Systems with State Delay and Parameter Uncertainty. IEEE Transactions on Automatic Control, 47, 1122-1228. http://dx.doi.org/10.1109/TAC.2002.800651 
[17] Fridman, E. (2002) Stability of Linear Descriptor Systems with Delay: A Lyapunov Based Approach. Journal of Mathematical Analysis and Applications, 273, 24-44. http://dx.doi.org/10.1016/S0022-247X(02)00202-0

[18] Park, P. and Ko, J.W. (2007) Stability and Robust Stability for Systems with a Time-Varying Delay. Automatica, 43, 1855-1858. http://dx.doi.org/10.1016/j.automatica.2007.02.022

[19] Han, Q.L. (2009) A Discrete Delay Decomposition Approach to Stability of Linear Retarded and Neutral Systems. Automatica, 45, 517-524. http://dx.doi.org/10.1016/j.automatica.2008.08.005

[20] Fridman, E., Shaked, U. and Liu, K. (2009) New Conditions for Delay-Derivative-Dependent Stability. Automatica, 45, 2723-2727. http://dx.doi.org/10.1016/j.automatica.2009.08.002

\section{Submit or recommend next manuscript to SCIRP and we will provide best service for you:}

Accepting pre-submission inquiries through Email, Facebook, Linkedin, Twitter, etc A wide selection of journals (inclusive of 9 subjects, more than 200 journals)

Providing a 24-hour high-quality service

User-friendly online submission system

Fair and swift peer-review system

Efficient typesetting and proofreading procedure

Display of the result of downloads and visits, as well as the number of cited articles

Maximum dissemination of your research work

Submit your manuscript at: http://papersubmission.scirp.org/ 